\title{
Pentosidine, advanced glycation end product, in acute ischaemic stroke patients with and without atrial rhythm disturbances
}

\author{
Marta Leńska-Mieciek ${ }^{1}$, Grażyna Korczak-Kowalska ${ }^{2,3}$, Katarzyna Bocian², Urszula Fiszer ${ }^{1}$ \\ ${ }^{1}$ Department of Neurology and Epileptology, Centre of Postgraduate Medical Education, Warsaw, Poland \\ ${ }^{2}$ Department of Immunology, Faculty of Biology, University of Warsaw, Warsaw, Poland \\ ${ }^{3}$ Department of Clinical Immunology, Transplantation Institute, Medical University of Warsaw, Warsaw, Poland
}

\begin{abstract}
Atrial fibrillation (AF) and atherosclerotic disease are independent risk factors for acute ischaemic stroke (AIS). The optimal biological marker which could allow differentiation between AF and non-AF AIS patients is still not available.

Aim of the study. Aim of the present study was to investigate the role of pentosidine as a potential biological marker for AF in an AIS patient group.

Materials and methods. Sixty-three acute ischaemic hemispheric stroke patients were recruited and divided into two groups according to the presumed underlying mechanism: with or without atrial rhythm disorders. Ten healthy volunteers were a reference group for serum level of pentosidine. Carotid artery ultrasound was performed, and common carotid artery stiffness and intima-media thickness were measured. Serum levels of pentosidine and selected routine biochemical risk factors for atherosclerosis (cholesterol and its lipoprotein fractions, homocysteine) were examined.

Results. A higher serum level of pentosidine was observed in patients without atrial fibrillation $(1,509 \pm 485.13 \mathrm{pmol} / \mathrm{ml})$; a statistically significant difference was observed compared to the reference group $(1,041.52 \pm 411.17 \mathrm{pmol} / \mathrm{ml} ; \mathrm{p}=0.01)$, but not the AF patients $(1,438.19 \pm 495.97 \mathrm{pmol} / \mathrm{ml} ; \mathrm{p}=0.59)$. No significant difference in the non-AF group compared to the AF group for carotid intima-media thickness (IMT)/stiffness and pentosidine serum level was recorded.

Conclusions and clinical implications. A higher serum level of pentosidine was observed in AIS patients without atrial fibrillation compared to the healthy volunteers. According to the results of the present study, no difference between these patients in the selected risk factors of atherosclerosis were observed. Further studies are needed to identify a reliable marker of AF that would bring added value to the standard diagnostic workup after acute ischaemic stroke.
\end{abstract}

Key words: acute ischaemic stroke, atrial fibrillation, pentosidine, carotid ultrasound

(Neurol Neurochir Pol 2020; 54 (4): 323-328)

\section{Introduction}

Atrial fibrillation $(\mathrm{AF})$ and atherosclerotic disease are independent risk factors for acute ischaemic stroke (AIS) [1-3]. In $20-25 \%$ of AIS cases, no causative factor can be found in routine tests. There is a need for biomarkers that are capable of identifying them. In recent years, a number of trials have studied the potential biological markers of AF. Four groups can be differentiated: markers of inflammation (e.g. pentraxin-3, neutrophil-to-lymphocyte ratio); markers of fibrosis (e.g. galectin-3, TGF-b1, matrix metalloproteinase -9 , growth/differentiation factor -15 ); markers of hormonal activity (e.g. N-terminal prohormone, fibroblast growth factor - 23); and others (e.g. circulating procoagulant microparticles, asymmetric dimethylarginine, microRNA) $[4,5]$.

Address for correspondence: Marta Leńska-Mieciek, Department of Neurology and Epileptology, Centre of Postgraduate Medical Education, Warsaw, Poland, e-mail: martalenska@wp.pl 
Atherosclerosis is characterised by intramural thickening of the sub-intima, and therefore, the carotid intima-media thickness (IMT) is now used as a marker of subclinical atherosclerosis [6-11]. A popular hypothesis is that oxidative stress promotes atherogenesis by accelerating glycation, and leads to the accumulation of advanced glycation end products (AGEs), which increase tissue stiffness and reduce elasticity [12-14]. Pentosidine is a well-characterised natural AGE that is used as a biomarker for AGE production.

Finding an optimal, biological marker for AF would be of great importance for stroke patients.

\section{Aim of study}

The aim of the present study was to investigate the role of pentosidine as a potential biological marker for AF in an AIS patient group.

\section{Materials and methods}

Over 27 months, 73 participants were recruited into the study by neurologists working in the Department of Neurology. The study group included 63 patients with hemispheric AIS, all of whom were treated at the stroke unit, plus 10 healthy volunteers (reference group for serum level of pentosidine). Hemispheric stroke was diagnosed based on the hemispheric syndrome. Age-matched staff family members were enrolled as the healthy controls. The inclusion criteria to the study group were as follows: (1) a patient with AIS, who (2) was able to provide informed consent. The exclusion criteria were: (1) a diagnosis other than AIS responsible for the neurological syndrome and (2) a refusal or inability to give informed consent. The 63 stroke patients were divided into two groups, according to the presumed underlying mechanism i.e. with and without atrial rhythm disorders. AF was diagnosed in 22 patients (the AF group). Patients were allocated to this group if there was a history of AF or if AF was observed during 24 hours of ECG monitoring (a standard test during hospitalisation). The 41 remaining stroke patients, who did not have heart rhythm disorders, were assigned to the non-AF group.

Carotid artery ultrasound was performed using the MYLAB 70 platform, with linear array transducer LA 522. IMT was measured at the posterior wall of the right and left CCAs, $10 \mathrm{~mm}$ from the carotid bifurcation. CCA-IMT was measured with Mylab 70 (Esaote) using the software-guided technique RF-Quality Intima Media Thickness (QIMT, Esaote). A region of interest was superimposed on the B-mode image. The mean of the IMT values was continuously calculated by the system and displayed on the left side of the image. The IMT values were expressed in micrometres. An example of the CCA-IMT measurement is shown in Figure 1. Common carotid artery stiffness was assessed using the Automatic Quality Arterial Stiffness (QAS) calculation, measuring the modification of arterial diameter between systolic and diastolic phases. The distensibility coefficient (DC), compliance coefficient (CC; ratio between variations in arterial cross-sectional area and blood pressure), and pulse wave velocity [PWV - time taken for a pressure pulse to travel between two set points] kPA-1 were calculated. The a and $\beta$ stiffness parameters were calculated as follows:

$$
\alpha=\mathrm{A} \mathrm{x} \ln (\mathrm{ps} / \mathrm{pd}) / \Delta \mathrm{A},
$$

where $\mathrm{A}$ - diastolic area, $\Delta \mathrm{A}$ - change of area in systole, ps - systolic pressure, pd - diastolic pressure); and

$$
\beta=\mathrm{D} \times \ln (\mathrm{ps} / \mathrm{pd}) / \Delta \mathrm{D},
$$

where $\mathrm{D}$ - diastolic diameter, $\Delta \mathrm{D}$ - change of diameter in systole, ps - systolic pressure, pd - diastolic pressure [15, 16].

All measurements of carotid stiffness were performed in the supine position by the same experienced neurosonologist (M.L.), both on the right and left common carotid arteries.

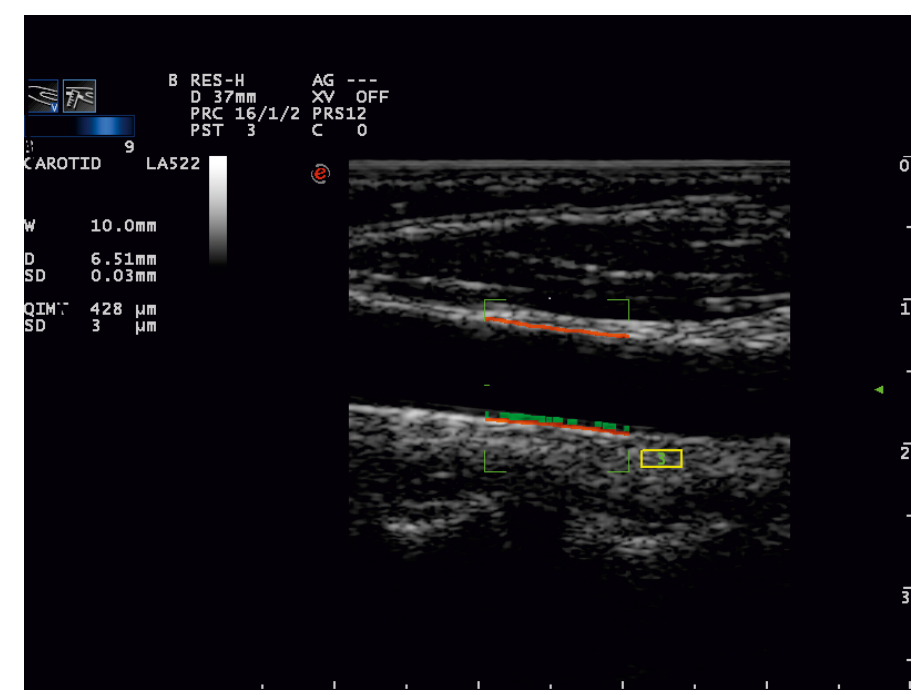

Figure 1. Common carotid artery intima-media thickness measurement using software-guided RF-Quality Intima Media Thickness (QIMT, Easote) 
For each patient, the systolic and diastolic brachial pressure was specified.

Routine biochemical risk factors for atherosclerosis, including the blood serum level of cholesterol and its lipoprotein fractions (high- and low-density lipoprotein; HDL and LDL, respectively), homocysteine and pentosidine were examined. All serum-level parameters were measured using standard laboratory practices within 72 hours of the onset of ischaemic stroke. The serum level of pentosidine was measured using a Human Pentosidine ELISA Kit (CUSABIO, Wuhan, China).

Characteristics of the study population were recorded and compared using the Student's t-test, Mann-Whitney U test, Kendall's tau-b test and Pearson's chi-squared test. All statistical analyses were performed using PAWS Statistic 18 (SPSS). $\mathrm{P}<0.05$ was considered statistically significant.

The local Ethical Committee approved the study.

\section{Results}

A total of 31 men and 32 women participated in the study: 22 AF patients (six men and 16 women; mean age: 76.82 years; age range: 61 to 90 ), and 41 non-AF patients (25 men and 16 women; mean age: 72.49 years; age range: 44 to 91 ). Four men and six women were included in the reference group (mean age: 71.8 years; age range: 66 to 78 ). The characteristics of each group are set out in Table 1.

A higher serum level of pentosidine was reported in the non-AF patients. A statistically significant difference compared to the reference group $(\mathrm{p}=0.01$ ), but not to the AF patients, was observed.

Although the distribution of males and females differed across the AF and non-AF groups, no statistically significant difference between men and women, in terms of any of the measured parameters, was observed within the groups. A higher serum level of triglycerides in men in the non-AF, compared to the AF, group was observed ( $\mathrm{p}=0.008$ ), but this was not the case for women. In both the AF and non-AF patient groups, the level of HDL cholesterol was higher $(p=0.016)$ and the CRP was lower $(\mathrm{p}=0.04)$ in women than in men (Tab. 2).

No statistically significant difference in the non-AF group compared to the AF group for DC, CC, PWV, IMT, $\alpha$ and $B$ stiffness and pentosidine serum level was recorded. Total cholesterol, triglycerides and LDL cholesterol serum levels tended to be higher in the non-AF group compared to the AF group, but a statistically significant difference between the two was recorded only for triglycerides $(\mathrm{p}=0.034)$.

No correlation between DC, CC, PWV, $\alpha$ and $B$ stiffness and pentosidine serum level was noted.

Ten out of 63 patients (16\%) suffered with diabetes mellitus (four in the AF group). Forty-four out of 63 (70\%) patients were diagnosed with arterial hypertension (13 in the AF group), and none with renal insufficiency. No statistically significant difference in pentosidine serum level between diabetes mellitus patients (mean: 1,601 $\pm 540 \mathrm{pmol} / \mathrm{ml}$ ) and patients without such a diagnosis (mean: $1,462 \pm 478 \mathrm{pmol} / \mathrm{ml}$; $\mathrm{p}=0.41)$ was recorded. No statistically significant difference in pentosidine serum level between patients with arterial hypertension (mean: $1,534 \pm 488 \mathrm{pmol} / \mathrm{ml}$ ) and patients without such a diagnosis (mean: $1,379 \pm 475 \mathrm{pmol} / \mathrm{ml}$; $\mathrm{p}=0.22)$ was noted.

There was no correlation between patient age and pentosidine serum level in the group of 63 patients $(p=0.70$, $r=-0.5)$, or in the AF group $(p=0.81, r=0.05)$, or in the non-AF group ( $\mathrm{p}=0.64, \mathrm{r}=-0.07)$.

\section{Discussion}

The present study provides a comparison of pentosidine serum level in non-AF and AF AIS patients. Little is known about the relationship between AGEs and AIS. A high serum level of pentosidine has been previously shown to be indicative of a poor prognosis 30 days after acute stroke and to be associated with branch atheromatous disease among small vessels occlusion $[17,18]$.

According to the results of the present study, no difference in serum level of pentosidine in non-AF and AF patients was noted. A higher serum level of pentosidine was reported in the non-AF patients, but there was a statistically significant difference compared only to the relatively small reference group $(p=0.01)$. There was no significant difference in the level of pentosidine between AF and healthy controls, but this is likely to have been caused by the sample size. The point estimates and $\mathrm{SD}$ in $\mathrm{AF}$ and non-AF groups are very similar. The $\mathrm{p}$ value of 0.06 indicates a clear tendency. Recent studies have shown higher subcutaneous content of AGEs in patients with subclinical atheromatous disease than in subjects without disease. No difference in serum level of pentosidine was reported between patients with and patients without generalised atheromatous disease [19]. Further studies are necessary to explore this for the AIS patients, and to establish reference values for serum level pentosidine in healthy controls.

The results of the present study showed no difference in carotid artery stiffness/IMT in AIS patients with and without AF. This confirms previous reports that increased IMT is associated with an increased stroke risk in AF patients [20]. Common carotid IMT, in the absence of cardiovascular risk factors, is strongly related to age [21]. In the present study, the age of patients in both groups was comparable. Increased carotid IMT is associated also with blood pressure variability, obesity and overweight $[22,23]$. These factors were not analysed in our study and they could interfere with the results. Mean IMT has been shown to be significantly correlated with systemic atherosclerotic change and PWV to predict the onset of stroke in hypertensive patients [24]. That was not confirmed in the present study, but hypertension was not an exclusion criterion in our study and patients with hypertension were included both in the AF and the non-AF groups. No difference in carotid artery stiffness/IMT in AIS patients with 


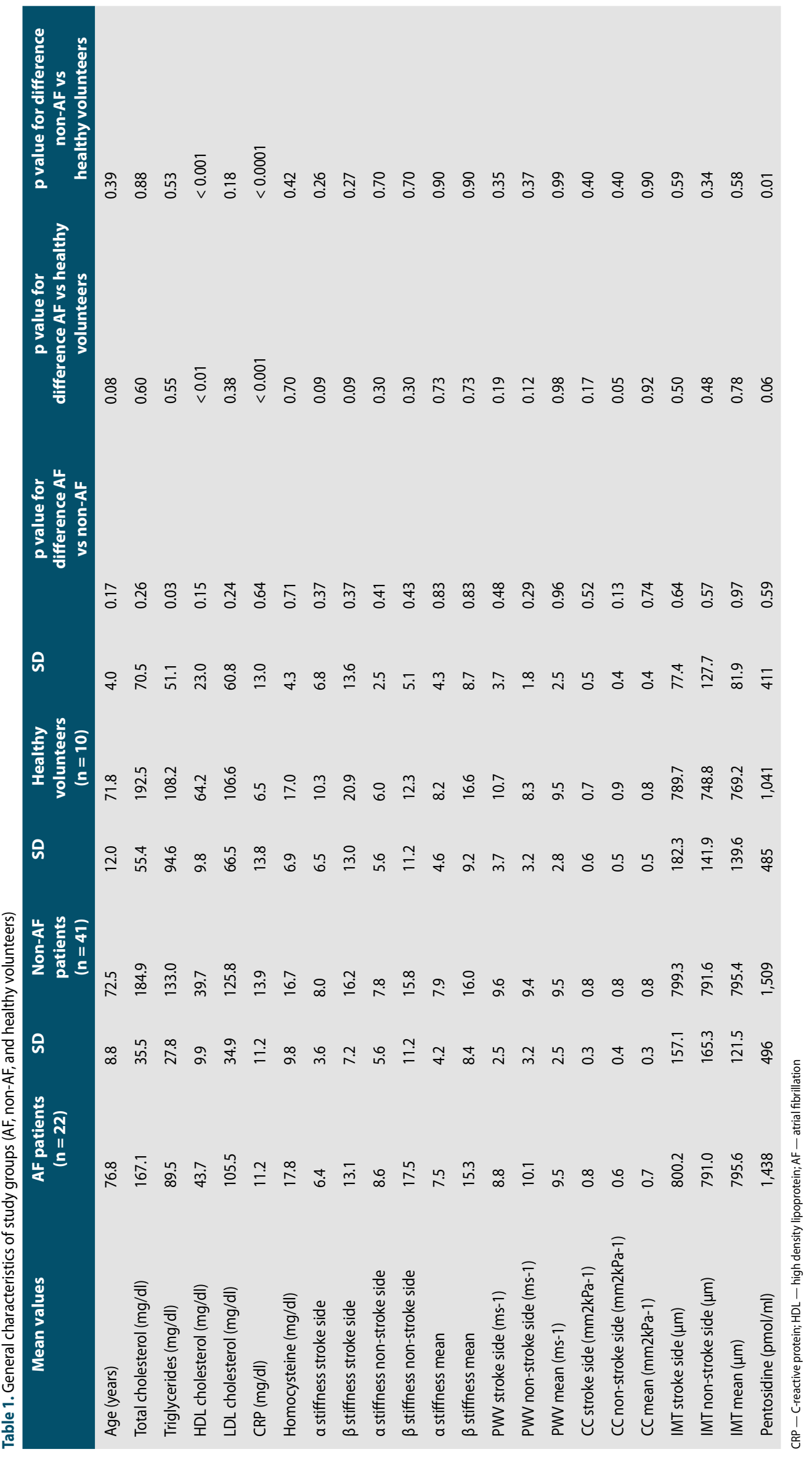




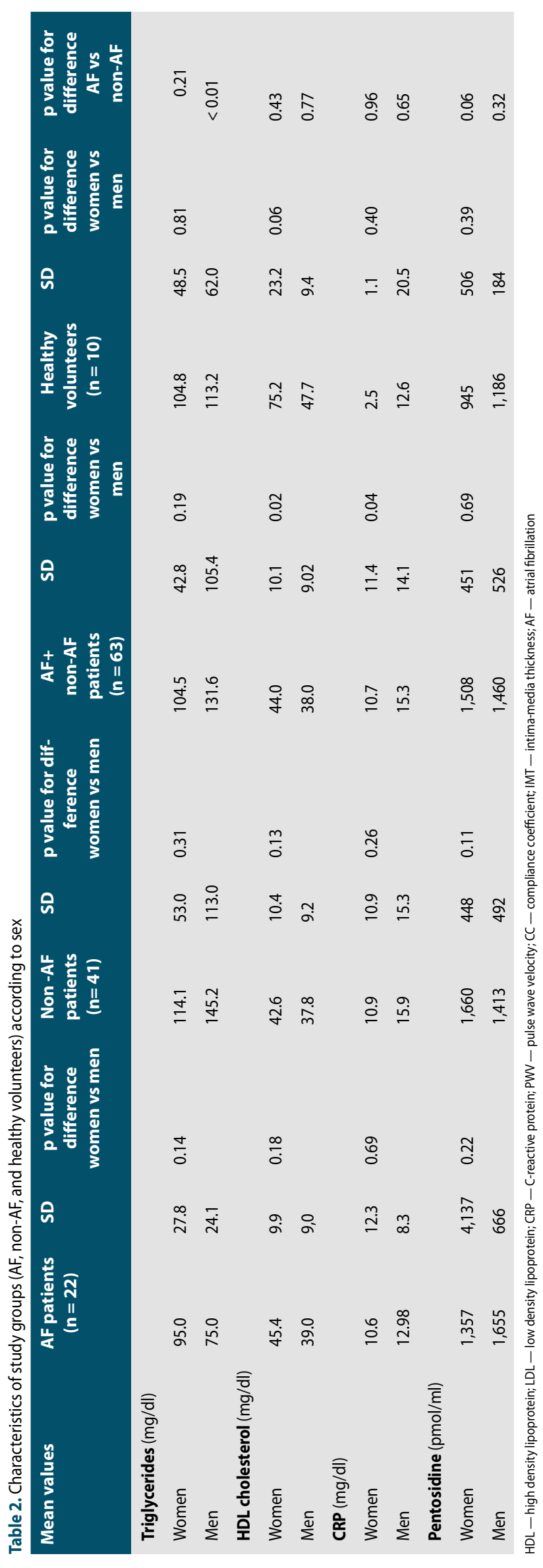

and without AF, as shown in the present study, supports the previous reports that higher carotid IMT and greater arterial stiffness are associated with higher AF incidence, and play a role in $\mathrm{AF}$ aetiopathogenesis [25].

Although several interesting and potentially important relationships have been established in the present study, it does face some limitations: a relatively small group of patients, an overrepresentation of non-AF stroke patients, an uneven sex distribution in groups, and the method of AF/non-AF group allocation. The exclusion criterion "refusal or inability to give informed consent" was responsible for the relatively low number of patients recruited to the study. All patients with aphasia and critical illness, who were unable to provide informed consent, were excluded from the study. Currently, only $1 / 6$ cases of stroke is attributed to AF. This is the reason for the overrepresentation of non-AF stroke patients in our study. Patients were allocated to the AF group either if there was a history of AF or if AF was registered during 24 hours of ECG monitoring. The detection of AF depends on the time of ECG monitoring [26], and is five times higher in 30-day event-triggered recording than when conventional 24-hour monitoring is used. One method of AF screening is an external miniaturised recorder with an adhesive electrode. This method enables the detection of AF in $12.3 \%$ of patients with stroke of unknown source during four weeks of monitoring [27]. This means that, potentially, in five patients from the presented non-AF group, AF would be revealed during four weeks of ECG monitoring. Implantable cardiac monitors may be recommended for some patients with AIS. Precise criteria for that procedure could enable the detection of AF in approximately $30 \%$ of patients [28].

\section{Conclusions}

Serum level of pentosidine was higher in non-AF patients, but it did not differentiate between AF and non-AF patients.

Cholesterol/LDL and carotid IMT correlate in patients without atrial fibrillation.

There was no difference in risk factors of atherosclerosis in the AF group compared to the non-AF group.

Funding source: This study was financially supported by the Center of Postgraduate Medical Education, Warsaw, Poland (project number 501-1-14-16-13). The funding body had no role in the design or conduct of the study, nor in the collection, management, analysis, or interpretation of the data. The funding body had no involvement in the preparation, review, or approval of the manuscript in which the findings of the study were reported. Conflicts of interest: None

\section{References}

1. Deb P, Sharma S, Hassan KM. Pathophysiologic mechanisms of acute ischemic stroke: An overview with emphasis on therapeutic significan- 
ce beyond thrombolysis. Pathophysiology. 2010; 17(3): 197-218, doi: 10.1016/j.pathophys.2009.12.001, indexed in Pubmed: 20074922.

2. Wolf PA, Abbott RD, Kannel WB. Atrial fibrillation as an independent risk factor for stroke: the Framingham Study. Stroke. 1991; 22(8): 983-988, doi: 10.1161/01.str.22.8.983, indexed in Pubmed: 1866765.

3. Falk E. Pathogenesis of Atherosclerosis. Journal of the American College of Cardiology. 2006; 47(8): C7-C12, doi: 10.1016/j. jacc.2005.09.068.

4. Szegedi I, Szapáry L, Csécsei P, et al. Potential Biological Markers of Atrial Fibrillation: A Chance to Prevent Cryptogenic Stroke. Biomed Res Int. 2017; 2017: 8153024, doi: 10.1155/2017/8153024, indexed in Pubmed: 28785588.

5. Ardhianto P, Yuniadi Y. Biomarkers of Atrial Fibrillation: Which One Is a True Marker? Cardiol Res Pract. 2019; 2019: 8302326, doi: 10.1155/2019/8302326, indexed in Pubmed: 31061732.

6. Bonomini F, Tengattini S, Fabiano A, et al. Atherosclerosis and oxidative stress. Histol Histopathol. 2008; 23(3): 381-390, doi: 10.14670/ HH-23.381, indexed in Pubmed: 18072094.

7. Cowdry EV. Arteriosclerosis: A survey of problem. New York: MacMillan; 1933 [Introduction.

8. Crowther MA. Pathogenesis of atherosclerosis. Hematology Am Soc Hematol Educ Program. 2005: 436-441, doi: 10.1182/asheducation-2005.1.436, indexed in Pubmed: 16304416.

9. O'Leary DH, Bots ML. Imaging of atherosclerosis: carotid intima-media thickness. Eur Heart J. 2010; 31(14): 1682-1689, doi: 10.1093/ eurheartj/ehq185, indexed in Pubmed: 20542989.

10. Stein JH, Korcarz CE, Hurst RT, et al. American Society of Echocardiography Carotid Intima-Media Thickness Task Force. Use of carotid ultrasound to identify subclinical vascular disease and evaluate cardiovascular disease risk: a consensus statement from the American Society of Echocardiography Carotid Intima-Media Thickness Task Force. Endorsed by the Society for Vascular Medicine. J Am Soc Echocardiogr. 2008; 21(2): 93-111; quiz 189, doi: 10.1016/j. echo.2007.11.011, indexed in Pubmed: 18261694.

11. Bruno RM, Bianchini E, Faita F, et al. Intima media thickness, pulse wave velocity, and flow mediated dilation. Cardiovasc Ultrasound. 2014; 12: 34, doi: 10.1186/1476-7120-12-34, indexed in Pubmed: 25148901.

12. Li H, Horke S, Förstermann U. Vascular oxidative stress, nitric oxide and atherosclerosis. Atherosclerosis. 2014; 237(1): 208-219, doi: 10.1016/j. atherosclerosis.2014.09.001, indexed in Pubmed: 25244505.

13. Yamagishi Si, Maeda S, Matsui T, et al. Role of advanced glycation end products (AGEs) and oxidative stress in vascular complications in diabetes. Biochim Biophys Acta. 2012; 1820(5): 663-671, doi: 10.1016/j.bbagen.2011.03.014, indexed in Pubmed: 21440603.

14. Goldin A, Beckman JA, Schmidt AM, et al. Advanced glycation end products: sparking the development of diabetic vascular injury. Circulation. 2006; 114(6): 597-605, doi: 10.1161/CIRCULATIONAHA.106.621854, indexed in Pubmed: 16894049.

15. Cecelja M, Chowienczyk P. Role of arterial stiffness in cardiovascular disease. JRSM Cardiovasc Dis. 2012; 1(4), doi: 10.1258/ cvd.2012.012016, indexed in Pubmed: 24175067.

16. Kubozono T, Ohishi M. Prognostic Significance of Regional Arterial Stiffness for Stroke in Hypertension. Pulse (Basel). 2015; 3(2): 98 105, doi: 10.1159/000381795, indexed in Pubmed: 26587458.
17. Ikeda T, Maruyama $\mathrm{K}$, Ito N, et al. Serum pentosidine, an advanced glycation end product, indicates poor outcomes after acute ischemic stroke. J Stroke Cerebrovasc Dis. 2012; 21(5): 386-390, doi: 10.1016/j.jstrokecerebrovasdis.2010.10.004, indexed in Pubmed: 21111635.

18. Ikeda $\mathrm{T}$, Maruyama $\mathrm{K}$, Ito $\mathrm{N}$, et al. High serum pentosidine in branch atheromatous disease among small vessels occlusion. J Neurosurg Sci. 2019; 63(4): 388-393, doi: 10.23736/S0390-5616.16.036286, indexed in Pubmed: 26899301.

19. Sánchez E, Betriu À, Yeramian A, et al. ILERVAS project, ILERVAS Project:. Skin Autofluorescence Measurement in Subclinical Atheromatous Disease: Results from the ILERVAS Project. J Atheroscler Thromb. 2019; 26(10): 879-889, doi: 10.5551/jat.47498, indexed in Pubmed: 30842389.

20. Bekwelem W, Jensen PN, Norby FL, et al. Carotid Atherosclerosis and Stroke in Atrial Fibrillation: The Atherosclerosis Risk in Communities Study. Stroke. 2016; 47(6): 1643-1646, doi: 10.1161/STROKEAHA.116.013133, indexed in Pubmed: 27217511.

21. Gori N, Anania G, Stefani L, et al. Carotid Intima-Media Thickness in Master Athletes. Asian J Sports Med. 2015; 6(2): e22587, doi: 10.5812/asjsm.6(2)2015.22587, indexed in Pubmed: 26448832.

22. Chen $\mathrm{Y}$, Xiong $\mathrm{H}, \mathrm{Wu} \mathrm{D}$, et al. Relationship of short-term blood pressure variability with carotid intima-media thickness in hypertensive patients. Biomed Eng Online. 2015; 14: 71, doi: 10.1186/s12938015-0059-8, indexed in Pubmed: 26204889.

23. Schiel R, Beltschikow W, Radón S, et al. Increased carotid intima-media thickness and associations with cardiovascular risk factors in obese and overweight children and adolescents. Eur J Med Res. 2007; 12(10): 503-508, indexed in Pubmed: 18024257.

24. Kawai T, Ohishi M, Takeya Y, et al. Carotid plaque score and intima media thickness as predictors of stroke and mortality in hypertensive patients. Hypertens Res. 2013; 36(10): 902-909, doi: 10.1038/ hr.2013.61, indexed in Pubmed: 23823172.

25. Chen LY, Leening MJG, Norby FL, et al. Carotid Intima-Media Thickness and Arterial Stiffness and the Risk of Atrial Fibrillation: The Atherosclerosis Risk in Communities (ARIC) Study, Multi-Ethnic Study of Atherosclerosis (MESA), and the Rotterdam Study. J Am Heart Assoc. 2016; 5(5), doi: 10.1161/JAHA.115.002907, indexed in Pubmed: 27207996.

26. Gladstone DJ, Dorian P, Spring M, et al. EMBRACE Steering Committee and Investigators, EMBRACE Investigators and Coordinators. Atrial fibrillation in patients with cryptogenic stroke. N Engl J Med. 2014; 370(26): 2467-2477, doi: 10.1056/NEJMoa1311376, indexed in Pubmed: 24963566.

27. Lumikari TJ, Putaala J, Kerola A, et al. Continuous 4-week ECG monitoring with adhesive electrodes reveals $A F$ in patients with recent embolic stroke of undetermined source. Ann Noninvasive Electrocardiol. 2019; 24(5): e12649, doi: 10.1111/anec.12649, indexed in Pubmed: 31045315.

28. Farkowski MM, Karliński MA, Kaźmierczak J, et al. Statement by a Working Group conceived by the Polish National Consultants in Cardiology and Neurology addressing the use of implantable cardiac monitors in patients after ischaemic embolic stroke of undetermined source. Neurol Neurochir Pol. 2019; 53(3): 181-189, doi: 10.5603/PJNNS. a2019.0018, indexed in Pubmed: 31145466. 